\title{
Identification of the Inflow Zone of Unruptured Cerebral Aneurysms: Comparison of 4D Flow MRI and 3D TOF MRA Data
}

\author{
K. Futami, H. Sano, K. Misaki, M. Nakada, F. Ueda, and J. Hamada
}

\begin{abstract}
BACKGROUND AND PURPOSE: The hemodynamics of the inflow zone of cerebral aneurysms may be a key factor in coil compaction and recanalization after endovascular coil embolization. We performed 4D flow MR imaging in conjunction with 3D TOF MRA and compared their ability to identify the inflow zone of unruptured cerebral aneurysms.
\end{abstract}

MATERIALS AND METHODS: This series comprised 50 unruptured saccular cerebral aneurysms in 44 patients. Transluminal color-coded 3D MRA images were created by selecting the signal-intensity ranges on 3D TOF MRA images that corresponded with both the luminal margin and the putative inflow.

RESULTS: 4D flow MR imaging demonstrated the inflow zone and yielded inflow velocity profiles for all 50 aneurysms. In 18 of 24 lateral-projection aneurysms (75\%), the inflow zone was located distally on the aneurysmal neck. The maximum inflow velocity ranged from 285 to 922 mm/s. On 4D flow MR imaging and transluminal color-coded 3D MRA studies, the inflow zone of 32 aneurysms (64\%) was at a similar location. In $91 \%$ of aneurysms whose neck section plane angle was $<30^{\circ}$ with respect to the imaging section direction on $3 \mathrm{D}$ TOF MRA, depiction of the inflow zone was similar on transluminal color-coded 3D MRA and 4D flow MR images.

CONCLUSIONS: 4D flow MR imaging can demonstrate the inflow zone and provide inflow velocity profiles. In aneurysms whose angle of the neck-section plane is obtuse vis-a-vis the imaging section on 3D TOF MRA scans, transluminal color-coded 3D MRA may depict the inflow zone reliably.

ABBREVIATION: TC 3D MRA = transluminal color-coded 3D MRA

A lthough endovascular coil embolization has become a major tactic to address cerebral aneurysms, recanalization or recurrence, which may result in rebleeding, are important problems. Recanalization was reported in $6.1 \%-39.8 \%$ of patients who had undergone endovascular treatment, ${ }^{1-6}$ and a meta-analysis found that $20.8 \%$ of treated aneurysms recurred. ${ }^{3}$ The rate of rerupture after endovascular treatment for ruptured aneurysms has ranged from $0.11 \%$ to $5.3 \%,{ }^{1,4,6}$ and the rupture rate in the first year after coil embolization was reported as $2.5 \%{ }^{7}$ and $2.2 \%{ }^{8}$ Because hemodynamics acting on the aneurysmal inflow zone may play a key role in the development of coil compaction or recanalization after endovascular coil embolization, the aneurysmal inflow zone must

Received August 27, 2013; accepted after revision December 16.

From the Department of Neurosurgery (K.F.), Mattoh-Ishikawa Central Hospital, Ishikawa, Japan; and Departments of Neurosurgery (H.S., K.M., M.N., J.H.) and Radiology (F.U.), Kanazawa University School of Medicine, Ishikawa, Japan.

Please address correspondence to Kazuya Futami, MD, Department of Neurosurgery, Mattoh-Ishikawa Central Hospital, 3-8 Kuramitsu, Hakusan, 920-8588 Ishikawa, Japan; e-mail: kfutami@mattohp.com

http://dx.doi.org/10.3174/ajnr.A3877 be packed densely to preserve the durability of aneurysm obliteration and to prevent rerupture. ${ }^{9-15}$

The inflow through the aneurysmal neck into the dome can be seen on 3D TOF MRA images. ${ }^{13,16,17}$ Satoh et al, ${ }^{16,17}$ who used conventional 3D TOF MRA techniques to select threshold ranges based on the signal intensity of the volume-rendering data, determined the spatial signal-intensity distribution in aneurysms. They developed transluminal color-coded 3D MRA (TC 3D MRA) to improve visualization of the aneurysmal inflow. More recently, 4D flow MR imaging based on time-resolved 3D cine phase-contrast MR imaging techniques was used to evaluate the hemodynamics of cerebral aneurysms. ${ }^{18-27}$ However, 4D flow MR imaging requires additional time for data acquisition, and TC 3D MRA may be a convenient alternative to $4 \mathrm{D}$ flow MR imaging for identifying the aneurysmal inflow zone.

Here, we compared the ability of $4 \mathrm{D}$ flow MR imaging and TC 3D MRA to identify the inflow zone of cerebral aneurysms.

\section{MATERIALS AND METHODS}

The institutional review board of Mattoh-Ishikawa Central Hospital approved this study; prior informed consent was obtained from all patients. 
Table 1: Aneurysm location and consistency of findings

\begin{tabular}{|c|c|c|c|c|c|c|c|}
\hline \multirow[b]{2}{*}{ Location } & \multirow[b]{2}{*}{ Total } & \multicolumn{2}{|c|}{$\begin{array}{l}\text { Accordant } \\
\text { (Group 1) }\end{array}$} & \multicolumn{2}{|c|}{$\begin{array}{c}\text { Contralateral } \\
\text { (Group 2) }\end{array}$} & \multicolumn{2}{|c|}{$\begin{array}{l}\text { Undefined } \\
\text { (Group 3) }\end{array}$} \\
\hline & & & $P$ Value & & $P$ Value & & $P$ Value \\
\hline \multicolumn{8}{|l|}{ ICA } \\
\hline Cavernous & 2 & 1 & 1.0 (NS) & 0 & 1.0 (NS) & 1 & .329 (NS) \\
\hline Paraclinoid & 11 & 6 & .494 (NS) & 5 & $.004(S)$ & 0 & .093 (NS) \\
\hline ICA-PcomA & 8 & 6 & .694 (NS) & 1 & 1.0 (NS) & 1 & .666 (NS) \\
\hline Bifurcation & 1 & 0 & .36 (NS) & 0 & 1.0 (NS) & 1 & .220 (NS) \\
\hline AcomA & 8 & 7 & .231 (NS) & 0 & .580 (NS) & 1 & .666 (NS) \\
\hline MCA & 16 & 10 & 1.0 (NS) & 1 & .406 (NS) & 5 & .297 (NS) \\
\hline BA & 4 & 2 & .612 (NS) & 0 & 1.0 (NS) & 2 & .206 (NS) \\
\hline Total & 50 & 32 & & 7 & & 11 & \\
\hline
\end{tabular}

Note:-S indicates significant; NS, not significant by the Fisher exact test adjusted for the $P$ value threshold for each location; BA, basilar artery; AcomA, anterior communicating artery; PcomA, posterior communicating artery.

Table 2: Aneurysm parameters: correlation of group 1 and group 2 and 3 aneurysms ${ }^{\mathrm{a}}$

\begin{tabular}{|c|c|c|c|c|c|c|}
\hline \multirow[b]{2}{*}{ Parameters } & \multirow[b]{2}{*}{ Total } & \multirow{2}{*}{$\frac{\text { Accordant }}{\text { (Group 1) }}$} & \multicolumn{2}{|c|}{ Contralateral } & \multicolumn{2}{|c|}{ Undefined } \\
\hline & & & (Group 2) & $P$ Value & (Group 3) & $P$ Value \\
\hline MD & $5.2 \pm 2.4$ & $5.3 \pm 2.1$ & $5.6 \pm 2.5$ & .570 (NS) & $4.6 \pm 1.4$ & .823 (NS) \\
\hline ND & $4.1 \pm 1.7$ & $4.2 \pm 1.7$ & $4.3 \pm 1.7$ & .891 (NS) & $3.7 \pm 1.7$ & .372 (NS) \\
\hline MD/ND ratio & $1.3 \pm 0.5$ & $1.3 \pm 0.4$ & $1.3 \pm 0.3$ & .608 (NS) & $1.4 \pm 0.8$ & .616 (NS) \\
\hline Maximum perpendicular height & $4.1 \pm 1.3$ & $4.0 \pm 1.4$ & $4.2 \pm 1.2$ & .583 (NS) & $4.1 \pm 1.3$ & .911 (NS) \\
\hline Aspect ratio & $1.1 \pm 0.5$ & $1.0 \pm 0.3$ & $1.0 \pm 0.4$ & .91 (NS) & $1.3 \pm 0.7$ & .344 (NS) \\
\hline Maximum height & $4.3 \pm 1.4$ & $4.3 \pm 1.4$ & $4.4 \pm 1.3$ & .826 (NS) & $4.2 \pm 1.4$ & .824 (NS) \\
\hline Size ratio & $1.6 \pm 0.6$ & $1.7 \pm 0.7$ & $1.4 \pm 0.4$ & .360 (NS) & $1.6 \pm 0.5$ & .922 (NS) \\
\hline Angle of neck section to imaging section & $52.1 \pm 21.8$ & $47.8 \pm 22.9$ & $52.9 \pm 10.0$ & .375 (NS) & $64.0 \pm 20.8$ & $.045(S)$ \\
\hline Maximum inflow velocity & $577 \pm 164$ & $604 \pm 169$ & $449 \pm 159$ & .029 (S) & $583 \pm 136$ & $.721(\mathrm{NS})$ \\
\hline MRA signal intensity & $355 \pm 69.0$ & $356 \pm 64.6$ & $348 \pm 56.8$ & .752 (NS) & $356 \pm 91.6$ & .955 (NS) \\
\hline
\end{tabular}

Note:-NS indicates not significant by the comparison test adjusted for the $P$ value threshold for each parameter; MD, maximum diameter; ND, neck diameter. ${ }^{a}$ Data are means.

\section{Materials}

Our study included 50 unruptured saccular cerebral aneurysms (44 patients); 24 were lateral projection and 26 were terminal aneurysms. They were evaluated by 3D TOF MRA and 4D flow MR imaging techniques. Of the 50 aneurysms, 2 were located on the cavernous and 11 on the paraclinoid segment of the ICA; 8 were on the bifurcation of the ICA and the posterior communicating artery; 1 was on the ICA bifurcation; 8 were on the anterior communicating artery; 16 , on the MCA bifurcation; and 4, on the basilar artery bifurcation. The maximum diameters of the aneurysms and the neck were $5.2 \pm 2.4 \mathrm{~mm}$ (range, $2.5-15 \mathrm{~mm}$ ) and $4.1 \pm 1.7 \mathrm{~mm}$ (range, $1.5-11.3 \mathrm{~mm}$ ), respectively.

\section{MR Imaging}

MR images were obtained on a 1.5T scanner (Magnetom Avanto; Siemens, Erlangen, Germany) with a slew rate of $125 \mathrm{~T} / \mathrm{m} / \mathrm{s}$. We used an 8-channel head array coil. Volume datasets were acquired by using a 3D TOF sequence with flow compensation in all 3 orthogonal directions. The imaging parameters for 3D TOF MRA were the following: TR/TE/NEX, $35 \mathrm{~ms} / 7.15 \mathrm{~ms} /$ average 1; flip angle, $22^{\circ}$; FOV, $150 \times 123 \mathrm{~mm}$; z-coverage, $45.6 \mathrm{~mm}$; 0.6-mm thickness; 3 slabs; 30 sections per slab; slab interval, $-4.2 \mathrm{~mm}$; matrix, $256 \times 168(512 \times 336$ with zero-filling interpolation processing); voxel size, $0.59 \times 0.73 \times 0.6 \mathrm{~mm}(0.295 \times 0.365 \times 0.6$ $\mathrm{mm}$ with zero-filling); bandwidth, $87 \mathrm{~Hz} / \mathrm{px}$; imaging time, 4 minutes 53 seconds; transaxial direction.

The details of $4 \mathrm{D}$ flow MR imaging are described elsewhere. ${ }^{18-20}$ The imaging parameters were the following: TR/TE/ NEX, $33.05 \mathrm{~ms} / 5.63 \mathrm{~ms} /$ average 1; flip angle, $22^{\circ}$; FOV, $200 \times 200$

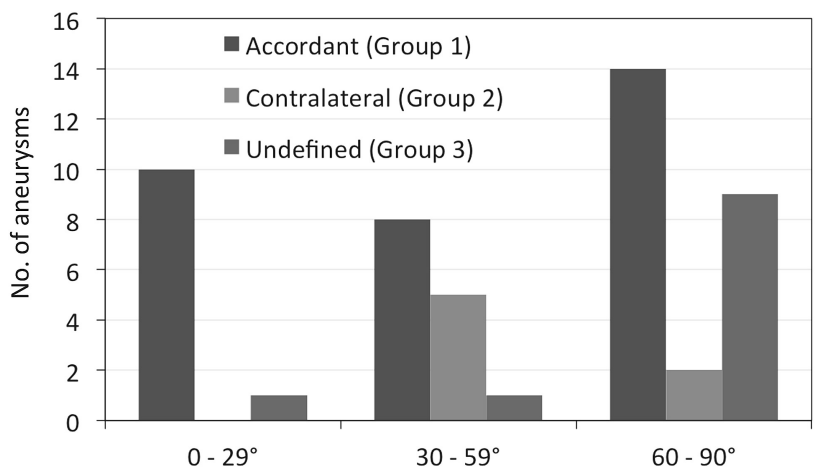

The angle of the section plane to imaging slice on 3D TOF MRA

FIG 1. The distribution of group 1,2 , and 3 aneurysms and the 3 ranges $\left(0^{\circ}-29^{\circ}, 30^{\circ}-59^{\circ}, 60^{\circ}-90^{\circ}\right)$ of the angle of the section plane that identified the aneurysm orifice with respect to the imaging section direction on 3D TOF MRA (group 1, the location of the inflow zone was in accord on 4D flow MR imaging and transluminal color-coded 3D MRA scans; group 2, the location of the inflow zone was on the contralateral side of the neck; group 3, the location of the inflow zone was undefined). There was a significant difference in the distribution among the angle ranges $(P=.018)$. Of 11 aneurysms with the $0^{\circ}-29^{\circ}$ angle range, $10(90.9 \%)$ were assigned to group 1 ; and the others, to group $3(P=.072$, compared with the other angle ranges).

mm; 0.8-mm thickness; 1 slab; $24-26$ sections per slab; z-coverage, $19.2 \mathrm{~mm}$; matrix, $192 \times 192$; no interpolation processing; voxel size, $1.04 \times 1.04 \times 0.8 \mathrm{~mm}$; velocity-encoding, $80 \mathrm{~cm} / \mathrm{s}$; bandwidth, $434 \mathrm{~Hz} / \mathrm{px}$; parallel imaging with reduction factor, 2 ; imaging time, 20-30 minutes depending on each patient's heart rate; transaxial direction; retrospective gating with electrocardiogram; 20 phases. 

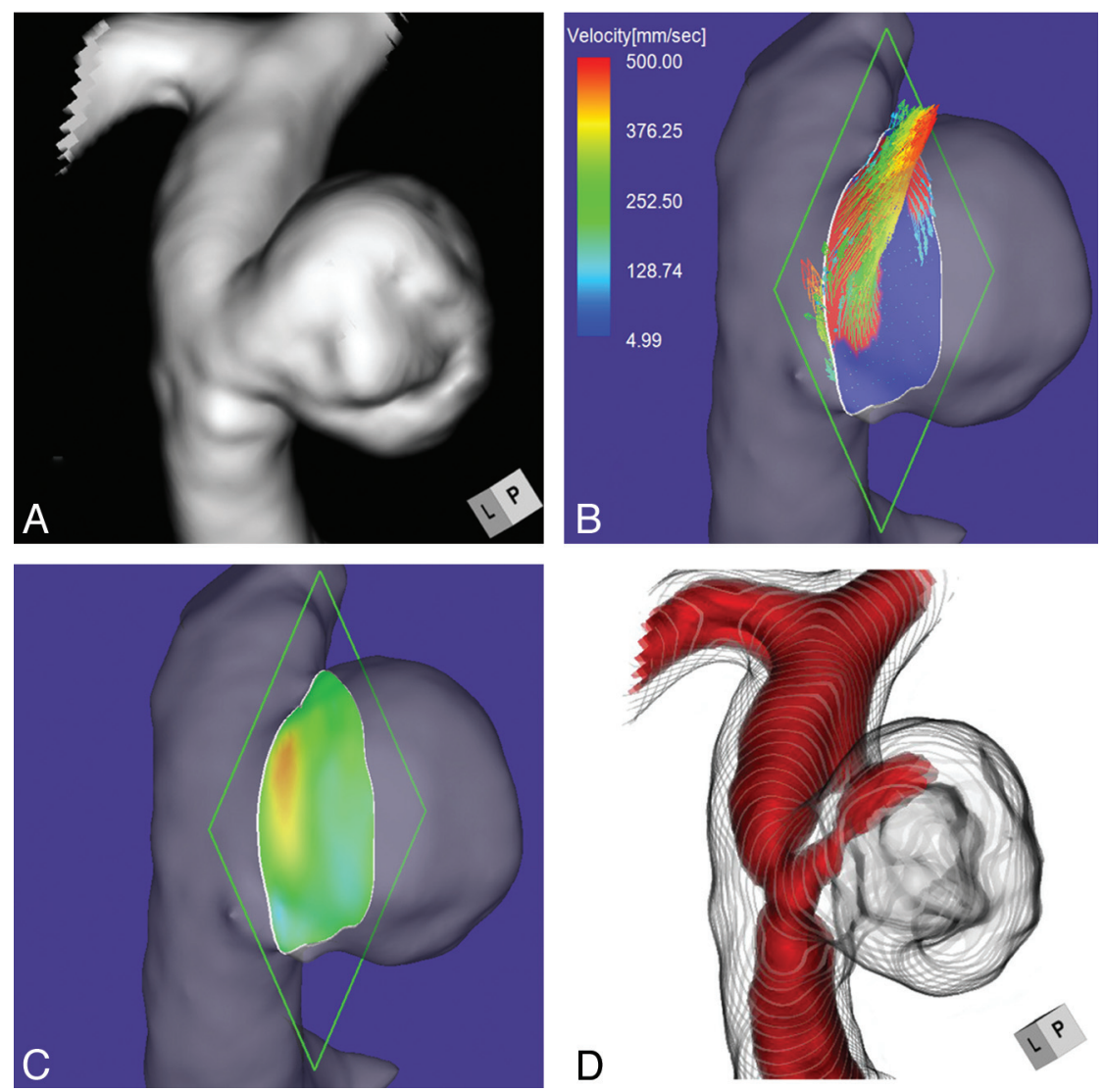

FIG 2. Case 1. A 75-year-old woman with an unruptured right ICA-posterior communicating artery bifurcation aneurysm. A, 3D TOF MRA image. B, 4D flow MR image demonstrates the inflow area (red) on the section plane and yields inflow velocity profiles. The angle of the section plane that determines the aneurysm orifice with respect to the imaging section direction on 3D TOF MRA is $73^{\circ}$. C, 4D flow MR image demonstrates the inflow zone (red). D, TC 3D MRA image depicts the putative inflow zone located on the distal neck. Red indicates the area where the MR signal intensity exceeded 375 .

The 3D datasets obtained by $4 \mathrm{D}$ flow MR imaging were analyzed with commercially available software (Flova II, Version 2.8.6; R'tech Co, Hamamatsu, Japan). On the basis of the 3D TOF MRA datasets, the vascular structures were segmented by using the region-growing method. ${ }^{28}$ Vascular shapes were created with the "marching cubes" method. ${ }^{29}$ For visualization of 3D flow information, the $3 \mathrm{D}$ datasets obtained by $4 \mathrm{D}$ flow MR imaging were converted to pixel datasets at a spatial resolution of $0.5 \times 0.5 \times$ $0.5 \mathrm{~mm}$ by a function of the software. To evaluate the inflow area, we selected a section plane corresponding to the aneurysmal orifice. Cerebral aneurysms with a complicated neck configuration were excluded from this study. Three of the authors (K.F., M.N., and F.U.) determined the window width and level of all datasets and selected the section plane of the neck. Each had $>15$ years of experience; decisions were made by consensus. The inflow zone obtained by $4 \mathrm{D}$ flow MR imaging was defined as the orifice area where components vertical to the section plane of the inflow vectors exceeded $60 \%$ of the maximum inflow velocity at peak systole; automatic depiction was as an animation image.

Details on the reconstruction of TC 3D MRA images have been described elsewhere. ${ }^{16,17}$ Briefly, volume data comprising 76 source axial images were transferred to a workstation with medical visualization software (Zio Station 2, Version 2.1; AMIN, Tokyo, Japan). Using a perspective volume-rendering algorithm, we created TC 3D MRA images by selecting a histogram of the signal intensity of volume-rendering 3D TOF MRA data that corresponded to the luminal margin (signal intensity; 145-160) and by determining the depiction range of the signal-intensity distribution in the aneurysmal lumen. On TC 3D MRA images, the luminal margin was visualized as a series of rings. The putative inflow zone on TC 3D MRA images was defined as the part of the orifice where the area of high MR signal intensity continued through the orifice into the aneurysmal lumen. The MR signal intensity used to identify the inflow zone ranged from 240 to 550 (mean; $355 \pm 69.0)$.

\section{Data Analysis}

We compared the inflow zone of the cerebral aneurysms identified by TC 3D MRA and $4 \mathrm{D}$ flow MR imaging. We also compared data obtained by these methods with respect to the aneurysm location, morphologic parameters, maximum inflow velocity, MRA signal intensity of the inflow, and angle of the section plane that identified the aneurysm orifice with respect to the imaging section direction on 3D TOF MRA. The morphologic parameters included the maximum diameter of the aneurysm and its neck, the maximum perpendicular height, the maximum height, the aspect ratio, and the size ratio of the aneurysm. ${ }^{30-32}$ Each numeric value of the various parameters was determined as the mean of the nearest 2 values independently estimated by the 3 readers.

Data are expressed as the mean \pm SD. For statistical analysis, we used the Student $t$ test or the Mann-Whitney $U$ test for continuous variables and the Fisher exact test for categoric variables. A $P$ value $<.05$ was considered significant.

\section{RESULTS}

$4 \mathrm{D}$ flow MR imaging demonstrated the inflow zone and the inflow velocity profiles. In 18 of the 24 lateral-projection aneurysms, the inflow zone was in the distal neck; in 2, it was in the proximal neck; and in 4, it was on the other side of the neck. In these 24 aneurysms, there was no significant difference with respect to their location or morphologic parameters. In all 26 terminal aneurysms, the inflow zone encompassed a line extending from the central axis of the parent artery. Maximum inflow velocity ranged from 285 to $922 \mathrm{~mm} / \mathrm{s}$ (mean, $577 \pm 164 \mathrm{~mm} / \mathrm{s}$ ).

In 32 aneurysms (group 1,64\%), the location of the inflow zone was in accord on 4D flow MR imaging and TC 3D MRA scans; in 7 (group 2,14\%), it was on the contralateral side of the neck, and in 11 (group 3, 22\%), its location was undefined. We looked for factors involved in the consistency of findings made by TC 3D MRA and 4D flow MR imaging with respect to the location of the 

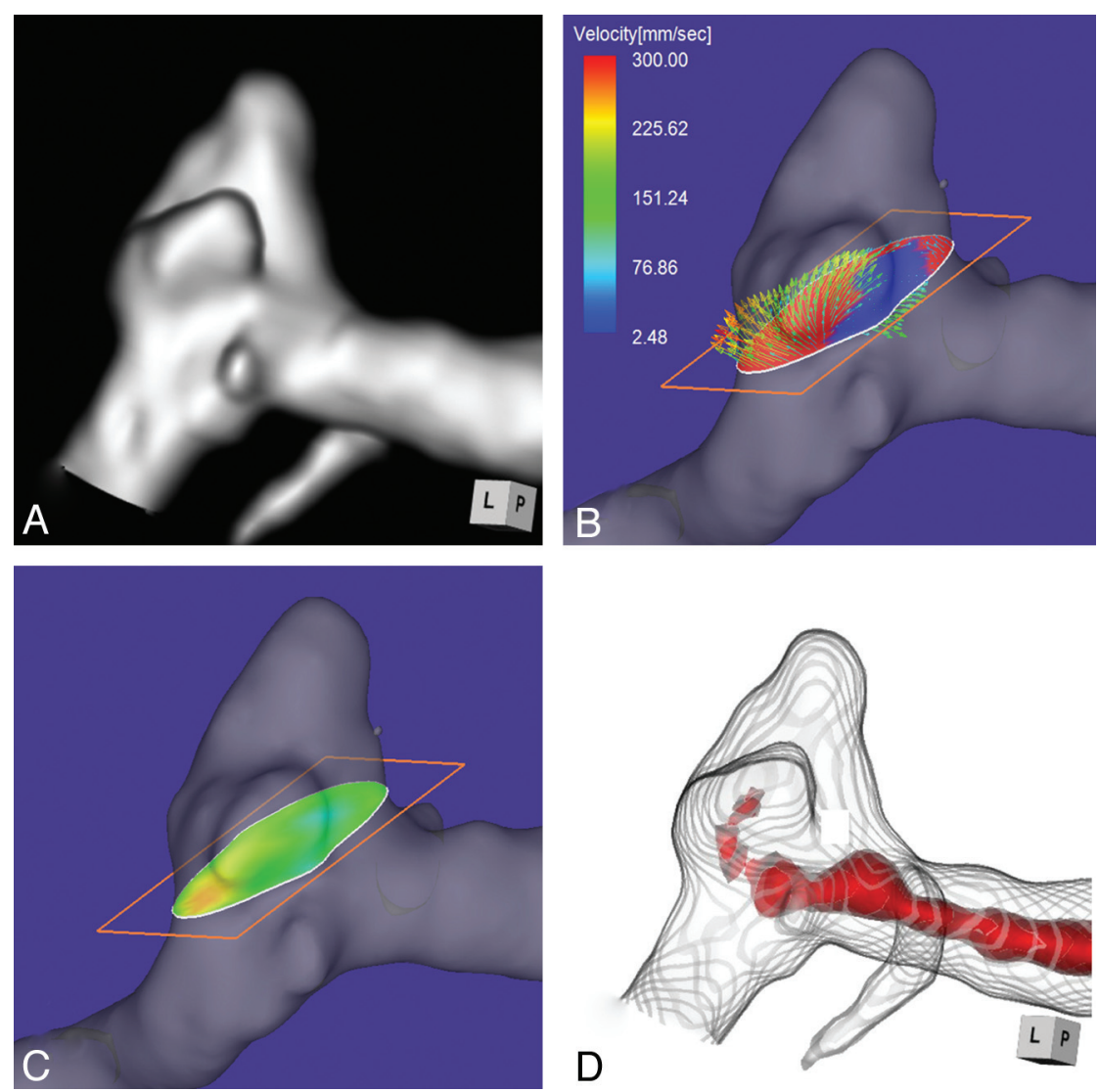

FIG 3. Case 2. A 58-year-old woman with an unruptured multilobulated aneurysm at the left MCA bifurcation. A, 3D TOF MRA. B, 4D flow MR imaging demonstrates inflow entering through the distal neck of the aneurysmal orifice. The angle of the section plane that identifies the aneurysmal orifice with respect to the imaging section direction on 3D TOF MRA is $25^{\circ}$. C, 4D flow MR imaging demonstrates the inflow zone (red). D, TC 3D MRA depicts the putative inflow zone located on the distal neck. Red indicates the area where the MR signal intensity exceeds 320 .

inflow zone (Tables 1 and 2). In 11 aneurysms on the paraclinoid segment of the ICA, there was a significant correlation with findings that located the inflow zone on the contralateral side of the neck $(P=$ .004). The maximum inflow velocity was significantly lower in group 2 than group 1 aneurysms ( $449 \pm 159$ versus $583 \pm 136 \mathrm{~mm} / \mathrm{s}, P=$ .029). There was no significant difference with respect to morphologic parameters and the MRA signal intensity.

The angle of the section plane of the neck with respect to the imaging section on 3D TOF MRA was significantly lower in group 1 than in group $3\left(47.8 \pm 22.8^{\circ}\right.$ versus $\left.64.0 \pm 20.8^{\circ}, P=.045\right)$ (Table 2). Figure 1 shows the distribution of group 1, 2, and 3 aneurysms and the degree ranges $\left(0^{\circ}-29^{\circ}, 30^{\circ}-59^{\circ}, 60^{\circ}-90^{\circ}\right)$ of the angle of the section plane with respect to the imaging section. The difference in the distribution among the 3 degree ranges was significant $(P=.018)$. Ten of 11 aneurysms with an angle range of $0^{\circ}-29^{\circ}(90.9 \%)$ were in group 1 ; the other was a group 3 aneurysm ( $P=.072$, compared with the other angle ranges).

\section{Case Presentation}

Case 1. Case 1 was a 75-year-old woman with an incidentally detected unruptured aneurysm at the right ICA-posterior communicating artery bifurcation. Its maximum diameter and neck diameter were 8.0 and $4.6 \mathrm{~mm}$, respectively (Fig $2 A$ ). The angle of the section plane that identified the aneurysm orifice with respect to the imaging section direction on $3 \mathrm{D}$ TOF MRA was $73^{\circ}$. $4 \mathrm{D}$ flow MR imaging demonstrated that the flow entered through the distal neck of the aneurysmal orifice (Fig 2B). At peak systole, the maximum inflow velocity was $536 \mathrm{~mm} / \mathrm{s}$. 4D flow MR imaging placed the inflow zone on the distal side of the orifice (Fig 2C). We used red color coding to identify the area where the MR signal intensity exceeded 375 on TC 3D MRA images (Fig $2 D)$. Consistent with the findings returned by $4 \mathrm{D}$ flow MR imaging, the inflow entered the aneurysmal lumen on the distal side of the neck. This aneurysm was placed in group 1 .

Case 2. Case 2 was a 58-year-old woman with an unruptured multilobulated aneurysm at the left MCA bifurcation. The aneurysmal maximum diameter and neck measured 5.1 and $5.0 \mathrm{~mm}$, respectively (Fig $3 A$ ). The angle of the section plane that identified the orifice with respect to the imaging section direction on 3D TOF MRA was $25^{\circ} .4 \mathrm{D}$ flow MR imaging showed that the inflow entered through the distal neck of the aneurysmal orifice (Fig 3B). The maximum inflow velocity was $301 \mathrm{~mm} / \mathrm{s}$. The inflow zone was located at the distal neck (Fig 3C). On TC 3D MRA images, the area with a signal intensity of $>320$ suggested that the inflow entered through the distal neck (Fig 3D). This aneurysm was recorded as a group 1 aneurysm.

Case 3. Case 3 was a 51-year-old woman who presented with a medially projecting unruptured aneurysm on the paraclinoid segment of the left ICA (Fig 4A). The maximum diameters of the aneurysm and the neck were 5.4 and $2.9 \mathrm{~mm}$, respectively. The angle of the section plane that identified the aneurysmal orifice with respect to the imaging section direction on 3D TOF MRA was $56^{\circ}$. $4 \mathrm{D}$ flow MR imaging showed that the inflow entered through a small area on the distal neck (Fig $4 B$ ) where the inflow zone was located (Fig 4C); maximum inflow velocity was $285 \mathrm{~mm} / \mathrm{s}$. On TC 3D MRA images, color coding of the area with a signal intensity of $>300$ suggested that the inflow entered through the proximal neck and flowed along the aneurysm wall (Fig 4D). Because these findings suggested that the putative inflow zone was located on the proximal rather than the distal neck, this aneurysm was placed in group 2 .

Case 4. Case 4 was a 70-year-old man who presented with a medially projecting, unruptured aneurysm on the paraclinoid segment of the right ICA (Fig 5A). The maximum diameters of the aneurysm and neck were 3.6 and $4.0 \mathrm{~mm}$, respectively. The angle of the section plane that identified the aneurysm orifice with respect to the imaging section direction on 3D TOF MRA 

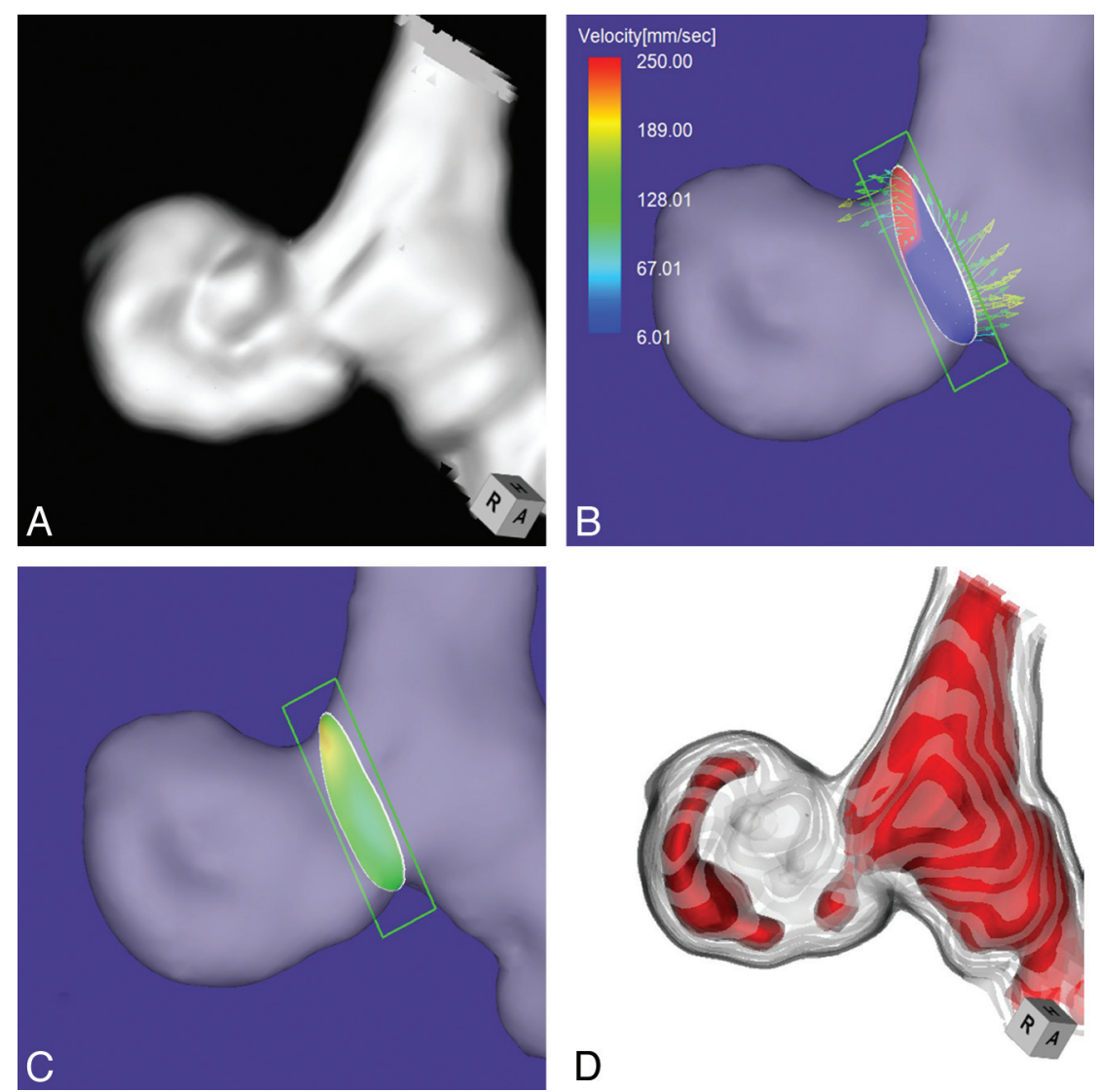

FIG 4. Case 3. A 51-year-old woman with a medially projecting unruptured aneurysm on the paraclinoid segment of the left ICA. A, 3D TOF MRA image. B, 4D flow MR imaging demonstrates flow entering through the distal neck. The angle of the section plane that identifies the aneurysm orifice with respect to the imaging section direction on 3D TOF MRA is $56^{\circ}$. C, 4D flow MR imaging shows the inflow zone (red). D, TC 3D MRA image depicts the putative inflow zone on the proximal neck. Red indicates the area where the MR signal intensity exceeds 300 .

was $65^{\circ} .4 \mathrm{D}$ flow MR imaging showed that the flow entered the aneurysmal lumen through an area along the distal margin of the neck (Fig 5B). The inflow zone was located on the distal neck (Fig 5C). The maximum inflow velocity was $406 \mathrm{~mm} / \mathrm{s}$. On TC 3D MRA images, color coding of the area with a signal intensity of $>395$ suggested that inflow entered through the proximal neck (Fig 5D). We posited that the putative inflow zone was located on the proximal rather than the distal neck and placed this aneurysm in group 2 .

Case 5. Case 5 was a 65 -year-old man with an unruptured lateralprojection aneurysm on the cavernous segment of the left ICA. The maximum diameters of the aneurysm and the neck were 7.3 and $6.8 \mathrm{~mm}$, respectively (Fig 6A). The angle of the section plane that identified the aneurysm orifice with respect to the imaging section direction on 3D TOF MRA was $86^{\circ}$. By 4D flow MR imaging, the inflow entered through the lateral half of the aneurysmal orifice (Fig $6 B$ ). The inflow zone was located on the side lateral to the neck (Fig 6C). The maximum inflow velocity was 647 $\mathrm{mm} / \mathrm{s}$. However, on TC 3D MRA images, identification of the area with a signal intensity of $>410$ suggested that the putative inflow zone was located on the distal neck (Fig 6D). This aneurysm was assigned to group 3.

\section{DISCUSSION}

Graves et $\mathrm{al}^{9}$ studied a canine carotid aneurysm model and reported that aneurysms exhibited 3 distinct flow zones: an inflow zone, an outflow zone, and a central slow flow vortex. They stressed the importance of packing the inflow zone for successful aneurysm obliteration. Coils placed in the inflow zone block the impact exerted by the blood stream on the aneurysmal wall and induce thrombosis formation within the dome. ${ }^{9,15}$ After endovascular treatment, the water hammer effect of the blood flow results in coil compaction in the inflow zone and recanalization. ${ }^{10,15}$ Valencia et al $^{14}$ observed high wall shear stress and increased pressure in the inflow zone; both are related to the regrowth and rerupture of cerebral aneurysms. Therefore, to obtain good treatment outcomes, one must identify the exact location of the inflow zone and evaluate the hemodynamics in this area.

During the coil embolization procedure, DSA may yield information on the temporally resolved hemodynamics and may help to identify the inflow zone. However, the information provided by this conventional method is limited. It cannot be used for the acquisition of quantitative variables of various hemodynamic parameters or of data at arbitrary time points in the cardiac cycle, and it is not 3D. Furthermore, DSA information can be affected by the speed or direction of the injected contrast medium and even by the mass effect of an inserted catheter.

$4 \mathrm{D}$ flow MR imaging facilitates the direct, noninvasive measurement of the in vivo $3 \mathrm{D}$ blood flow and its velocity in the vascular region of interest. ${ }^{33-35}$ Technologic advances in MR imaging have made it possible to assay the flow condition in aneurysms by $4 \mathrm{D}$ flow MR imaging. ${ }^{18,19}$ Both $4 \mathrm{D}$ flow MR imaging and computational fluid dynamics studies, used to evaluate the hemodynamics of cerebral aneurysms, returned similar results with respect to velocity distributions, inflow streamlines, and intraaneurysmal flow patterns in human cerebral ${ }^{19,21,23}$ and experimental canine aneurysms. ${ }^{36}$ In addition, 4D flow MR imaging results were validated by computational fluid dynamics studies performed on a life-size human aneurysm phantom. ${ }^{37-39}$ Moreover, previous studies confirmed the feasibility of depicting the aneurysmal inflow. ${ }^{18,20,23,26,27}$ Consequently, 4D flow MR imaging is the criterion standard for the identification of the inflow zone of cerebral aneurysms.

According to others, ${ }^{11,13,40}$ the inflow zone of lateral-projection aneurysms was located on the distal neck. However, in 6 of our 24 lateral-projection aneurysms (25\%), it was found on the other side of the neck. Because these 6 aneurysms did not differ in their location 

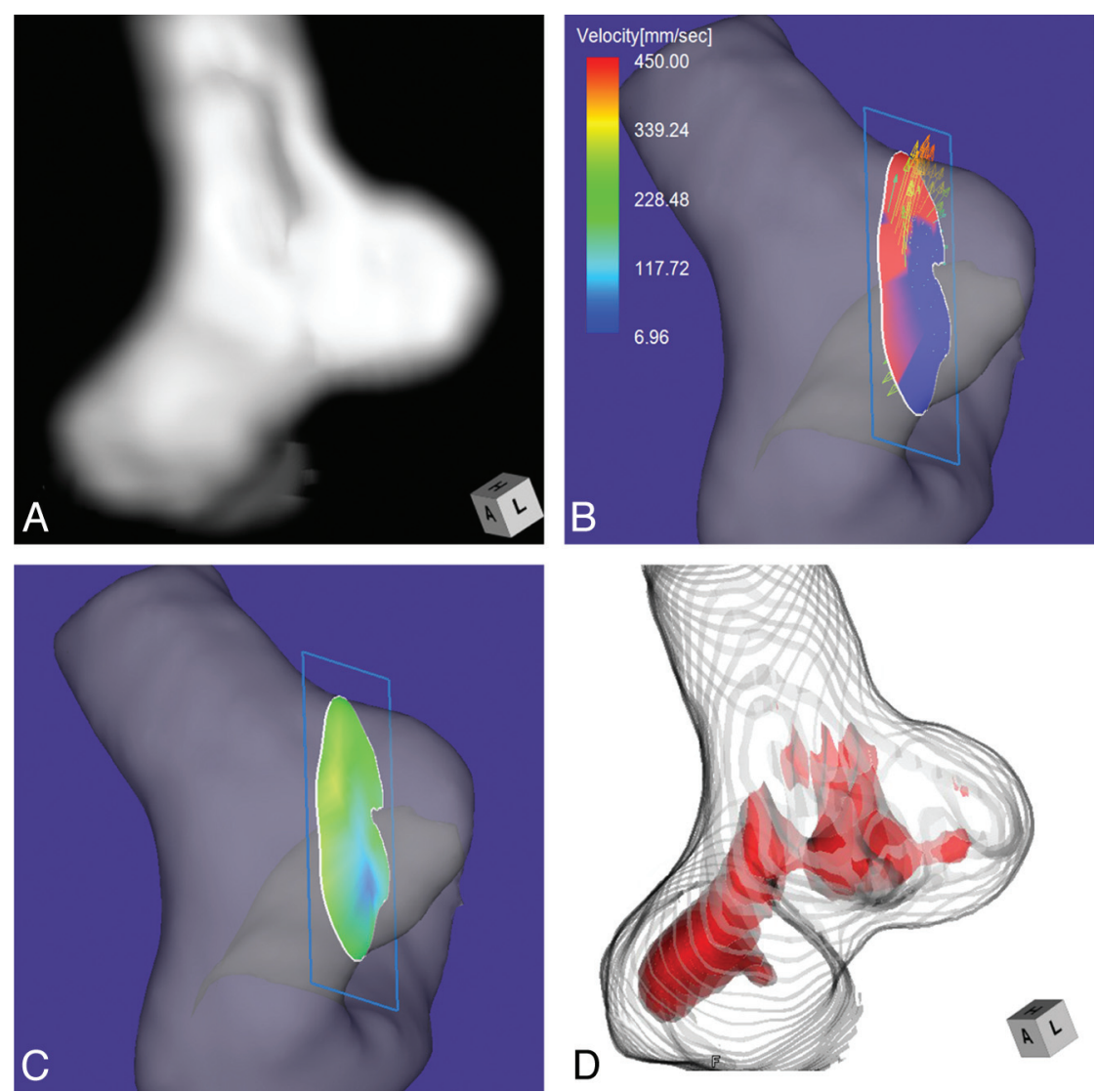

FIG 5. Case 4. A 70-year-old man with a medially projecting, unruptured aneurysm on the paraclinoid segment of the right ICA. A, 3D TOF MRA image. $B, 4 D$ flow MR image shows that the inflow enters through the area along the distal margin of the neck. The angle of the section plane that identifies the aneurysm orifice with respect to the imaging section direction on 3D TOF MRA is $65^{\circ}$. C, 4D flow MR imaging shows the inflow zone (yellow). D, TC 3D MRA image depicts the putative inflow zone on the proximal neck. Red indicates the area where the MR signal intensity exceeded 395.

and morphologic parameters from aneurysms whose inflow zone was on the distal neck, it is important to identify the exact location of the inflow zone even in lateral-projection aneurysms. The range of maximum inflow was very wide $(285-922 \mathrm{~mm} / \mathrm{s})$. High inflow velocity might be a predisposing factor for coil compaction or recanalization after endovascular coil embolization.

We investigated whether TC 3D MRA represents a convenient alternative to $4 \mathrm{D}$ flow $\mathrm{MR}$ imaging for identifying the aneurysmal inflow zone. We found that in $64 \%$ of our aneurysms (group 1), the inflow zone identified on TC 3D MRA and 4D flow MR imaging coincided, while in 14\%, especially in paraclinoid aneurysms, the 2 methods localized the inflow zone on opposite sides of the neck (group 2). To identify aneurysms whose inflow zone is reliably depicted on TC 3D MRA images without the aid of 4D flow MR imaging, we looked for factors that played a role in the consistency of findings made with TC 3D MRA and 4D flow MR imaging. Neither the aneurysm location, morphologic parameters, nor the MRA signal intensity of the inflow could predict the consistency.

The signal intensity of 3D TOF MRA is mainly affected by the flow velocity. ${ }^{41-44}$ In this study, the maximum inflow velocity in our group 2 aneurysms was significantly lower than that in group $1(P=.029)$. The sensitivity of 3D TOF MRA, on the other hand, is affected by the direction of the blood flow. ${ }^{41-44}$ We measured the angle of the section plane that identified the aneurysm orifice with respect to the imaging section direction on 3D TOF images. This angle was significantly smaller in group 1 than in group 3 aneurysms $(P=.045)$. In 10 of 11 aneurysms $(91 \%)$ whose angle ranged from $0^{\circ}$ to $29^{\circ}$, the inflow zone was at a similar location on 4D flow MR imaging and TC 3D MRA studies. In the remaining aneurysm, 3D TOF MRA failed to depict the putative inflow zone. Our findings suggest that if $3 \mathrm{D}$ TOF MRA can depict the putative inflow zone in aneurysms whose neck has a section plane angle below $30^{\circ}$ with respect to the imaging section direction, the information it yields is very precise. Concentric flow conditions such as slow or turbulent flow may lead to a regional elimination of TOF signal intensities, ${ }^{41-44}$ and this may result in misidentification of the inflow zone on 3D TOF MRA images. However, in this study, no aneurysms were excluded due to signal loss when estimating the inflow zone and measuring morphologic parameters. Moreover, in conjunction with interpolation methods for $3 \mathrm{D}$ TOF MRA, the voxel dimensions may affect TOF signal intensities. ${ }^{42-44}$ Further studies are needed to improve the accuracy of 3D TOF MRA results,

Our study has some limitations. First, because most of the aneurysms were part of a long-term follow-up study, many of the included small and wide-neck aneurysms were not candidates for endovascular coil embolization. Second, for 4D flow MR imaging to evaluate the inflow zone, we used a section plane that corresponded to the aneurysm orifice. This rendered difficult the 4D flow MR imaging of cerebral aneurysms whose neck configuration was complicated, and such aneurysms were excluded from the present study. Third, the spatial resolution of both $4 \mathrm{D}$ flow MR imaging and 3D TOF MRA is limited. Although we expected the visibility of the inflow zone of small aneurysms to be poor on both types of study, there was no significant difference in our ability to identify the inflow zone in groups categorized by the maximum diameter of the aneurysm (data not shown). On 4D flow MR images, the spatial averaging effect and the partial volume effect may result in incorrect flow estimation. ${ }^{18}$ To evaluate the flow condition in aneurysms with a minimum diameter of $2.0 \mathrm{~mm}$, a spatial resolution of $0.5 \mathrm{~mm}$ in the isotropic voxel dimensions may be desirable. ${ }^{37} \mathrm{Be}-$ cause the number of aneurysms included in this study was relatively small, larger series are needed to identify the role of aneurysmal size.

\section{CONCLUSIONS}

We recommend that before endovascular coil embolization, the exact location of the inflow zone be identified, even in 

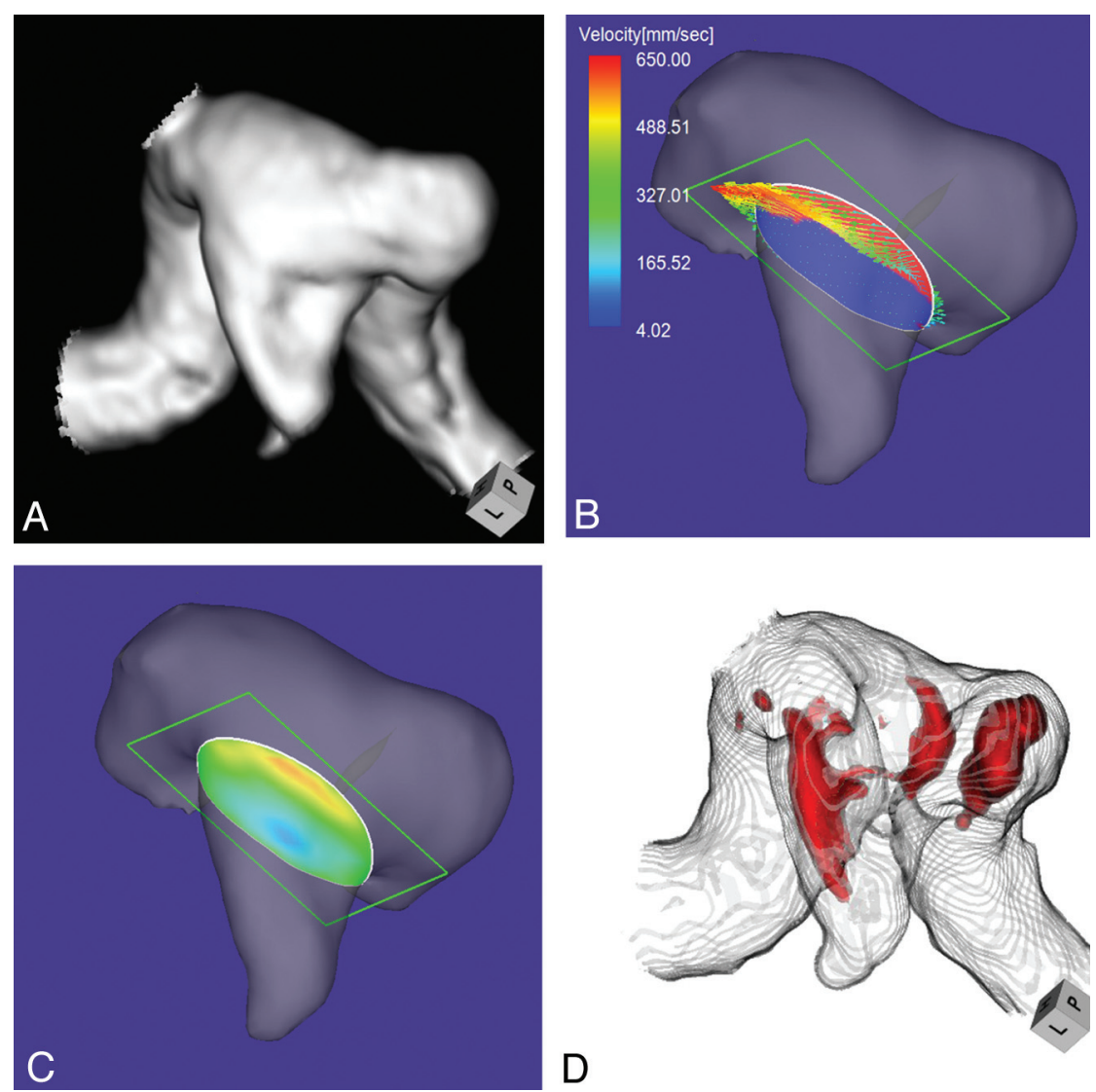

FIG 6. Case 5. A 65-year-old man with an unruptured aneurysm on the cavernous segment of the left ICA. A, 3D TOF MRA image. B, 4D flow MR image shows the inflow area (red) on the section plane and inflow velocity profiles. The angle of the section plane that identifies the aneurysm orifice with respect to the imaging section direction on 3D TOF MRA is $86^{\circ}$. C, 4D flow MR imaging shows the inflow zone (red). D, TC 3D MRA image depicts the putative inflow zone on the distal neck. Red indicates the area where the MR signal intensity exceeds 410.

lateral-projection aneurysms. 4D flow MR imaging can demonstrate the inflow zone and provide inflow velocity profiles. In aneurysms whose neck section plane angle is $<30^{\circ}$ with respect to the imaging section direction on 3D TOF MRA images, TC 3D MRA may depict the inflow zone reliably. Further studies on the hemodynamics of the inflow zone are needed to clarify the mechanisms of coil compaction or recanalization in patients with cerebral aneurysms treated by endovascular coil embolization.

\section{ACKNOWLEDGMENTS}

We are grateful to Professor Jun-ichiro Hamada for his encouragement and his dedication to our work. We mourn his untimely death. We thank Aki Watanabe, MR application specialist, Application Department, Siemens Japan, Tokyo, Japan, for technical advice, and Keiko Sumita, RT, and Takamitsu Matsuzaki, RT, of the Department of Radiation Technology, Mattoh-Ishikawa Central Hospital, Hakusan, Japan, for their dedicated support in performing this study.

\section{REFERENCES}

1. Choi DS, Kim MC, Lee SK, et al. Clinical and angiographic longterm follow-up of completely coiled intracranial aneurysms using endovascular technique. J Neurosurg 2010;112:575-81
2. Crobeddu E, Lanzino G, Kallmes DF, et al. Review of 2 decades of aneurysm-recurrence literature. Part 2. Managing recurrence after endovascular coiling. AJNR Am J Neuroradiol 2013;34:481-85

3. Ferns SP, Sprengers MES, van Rooji WJ, et al. Coiling of intracranial aneurysms: a systematic review on initial occlusion and reopening and retreatment rates. Stroke 2009;40:e523-29

4. Ries T, Siemonsen S, Thomalla G, et al. Long-term follow-up of cerebral aneurysms after endovascular therapy: prediction and outcome of retreatment. AJNR Am J Neuroradiol 2007;28:1755-61

5. Tso MK, Kochar P, Goyal M, et al. Longterm angiographic and clinical outcomes in completely versus incompletely coiled ruptured intracranial aneurysms. Clin Neurosurg 2011;58:143-48

6. Willinsky RA, Peltz J, da Costa L, et al. Clinical and angiographic follow-up of ruptured intracranial aneurysms treated with endovascular embolization. AJNR Am J Neuroradiol 2009;30:1035-40

7. Johnston SC, Dowd CF, Higashida RT, et al. Predictors of rehemorrhage after treatment of ruptured intracranial aneurysms: the Cerebral Aneurysm Rerupture After Treatment (CARAT) study. Stroke 2008;39:120-25

8. Molyneux A, Kerr R, Stratton I, et al, for the International Subarachnoid Aneurysm Trial (ISAT) Collaborative Group. International Subarachnoid Aneurysm Trial (ISAT) of neurosurgical clipping versus endovascular coiling in 2143 patients with ruptured intracranial aneurysms: a randomised trial. Lancet 2002;360:1267-74

9. Graves VB, Strother CM, Duf TA, et al. Early treatment of ruptured aneurysms with Guglielmi detachable coils: effect on subsequent bleeding. Neurosurgery 1995;37:640-48, discussion 647-48

10. Hayakawa M, Murayama Y, Duckwiler GR, et al. Natural history of the neck remnant of a cerebral aneurysm treated with the Guglielmi detachable coil system. J Neurosurg 2000;93:561-68

11. Hoh BL, Putman CM, Budzik RF, et al. Combined surgical and endovascular techniques of flow alteration to treat fusiform and complex wide-necked intracranial aneurysms that are unsuitable for clipping or coil embolization. J Neurosurg 2001;95:24-35

12. Imbesi SG, Kerber CW. Analysis of slipstream flow in a wide-necked basilar artery aneurysm: evaluation of potential treatment regimens. AJNR Am J Neuroradiol 2001;22:721-24

13. Kudo T, Satow T, Yamada N, et al. Identification of inflow zone of cerebral aneurysms by MRA for effective coil embolization [in Japanese with an English abstract]. Surg Cerebral Stroke (Jpn) 2008;36:459-63

14. Valencia AA, Guzmán AM, Finol EA, et al. Blood flow dynamics in saccular aneurysm models of the basilar artery. J Biomech Eng 2006;128:516-26

15. Viñuela F. Comments for histological findings in coil-packed experimental aneurysms 3 months after embolization. Neurosurgery 2002;50:384-85

16. Satoh T, Onoda K, Tsuchimoto S. Visualization of intraaneurysmal flow patterns with transluminal flow images of 3D MR angiograms in conjunction with aneurysmal configurations. AJNR Am J Neuroradiol 2003;24:1436-45

17. Satoh T, Ekino C, Ohsako C. Transluminal color-coded three-di- 
mensional magnetic resonance angiography for visualization of signal intensity distribution pattern within an unruptured cerebral aneurysm: preliminarily assessment with anterior communicating aneurysms. Neuroradiology 2004;46:628-34

18. Meckel S, Stalder AF, Santini F, et al. In vivo visualization and analysis of 3-D hemodynamics in cerebral aneurysms with flow-sensitized 4-D MR imaging at 3 T. Neuroradiology 2008;50:473-84

19. Boussel L, Rayz V, Martin A, et al. Phase-contrast magnetic resonance imaging measurements in intracranial aneurysms in vivo of flow patterns, velocity fields, and wall shear stress: comparisons with computational fluid dynamics. Magn Reson Med 2009;61:409-17

20. Hope TA, Hope MD, Purcell DD, et al. Evaluation of intracranial stenoses and aneurysms with accelerated 4D flow. Magn Reson Imaging 2010;28:41-46

21. Isoda H, Ohkura $\mathrm{Y}$, Kosugi $\mathrm{T}$, et al. Comparison of hemodynamics of intracranial aneurysms between MR fluid dynamics using 3D cine phase contrast MRI and MR-based computational fluid dynamics. Neuroradiology 2010;52:913-20

22. Isoda $\mathrm{H}$, Ohkura $\mathrm{Y}$, Kosugi $\mathrm{T}$, et al. In vivo hemodynamic analysis of intracranial aneurysms obtained by magnetic resonance fluid dynamics (MRFD) based on time-resolved three-dimensional phasecontrast MRI. Neuroradiology 2010;52:921-28

23. Naito T, Miyachi S, Matsubara N, et al. Magnetic resonance fluid dynamics for intracranial aneurysms: comparison with computed fluid dynamics. Acta Neurochir (Wien) 2012;154:993-1001

24. Kawaguchi T, Nshimura S, Kanamori M, et al. Distinctive flow pattern of wall shear stress and oscillatory shear index: similarity and dissimilarity in ruptured and unruptured cerebral aneurysm blebs. J Neurosurg 2012;117:774-80

25. Kecskemeti S, Johnson K, Wu Y, et al. High resolution three-dimensional cine phase contrast MRI of small intracranial aneurysms using a stack of stars k-space trajectory. J Magn Reason Imaging 2012;35:518-27

26. van Ooij P, Schneuders JJ, Marquering HA, et al. 3D cine phasecontrast MRI at $3 \mathrm{~T}$ in intracranial aneurysms compared with patient-specific computational fluid dynamics. AJNR Am J Neuroradiol 2013;34:1785-91

27. Schnell S, Ansari SA, Vakil P, et al. Three-dimensional hemodynamics in intracranial aneurysms: influence of size and morphology. $J$ Man Reason Imaging 2014;39:120-31

28. Lorensen WE, Cline HE. Marching cubes: a high resolution 3D surface construction algorithm. Comput Graph 1987;21:163-69

29. Shimai H, Yokota H, Nakamura S, et al. Extraction from biological volume data of a region of interest with nonuniform inten- sity. In Sumi K, ed. Optomechatronic Machine Vision. Proceedings of SPIE. Bellingham: WA: SPIE; 2005;6051:605

30. Dhar S, Tremmel M, Mocco J, et al. Morphological parameter for intracranial aneurysm rupture risk assessment. Neurosurgery 2008; 63:185-96, discussion 196-97

31. Raghavan ML, Ma B, Harbaugh RE. Quantified aneurysm shape and rupture risk. J Neurosurg 2005;102:355-62

32. Ujiie $\mathrm{H}$, Tamano $\mathrm{Y}$, Sasaki $\mathrm{K}$, et al. Is the aspect ratio a reliable index for predicting the rupture of a saccular aneurysm? Neurosurgery 2001;48:495-502, discussion 502-03

33. Pelc NJ, Herfkens RJ, Shimakawa A, et al. Phase contrast cine magnetic resonance imaging. Magn Reson Q 1991;4:229-54

34. Markl M, Chan FP, Alley MT, et al. Time-resolved three-dimensional phase-contrast MRI. J Magn Reason Imaging 2003;17:499-506

35. Bammer R, Hope TA, Aksoy M, et al. Time-resolved 3D quantitative flow MRI of the major intracranial vessels: initial experience and comparative evaluation at $1.5 \mathrm{~T}$ and $3.0 \mathrm{~T}$ in combination with parallel imaging. Magn Reson Med 2007;57:127-40

36. Jiang J, Johnson K, Valen-Sendstad K, et al. Flow characteristics in a canine aneurysm model: a comparison of $4 \mathrm{D}$ accelerated phase contrast MR measurements and computational fluid dynamics simulations. Med Phys 2011;38:6300-12

37. Hollnagel DI, Summers PE, Poulikakos D, et al. Comparative velocity investigations in cerebral arteries and aneurysms: 3D phasecontrast MR angiography, laser Doppler velocimetry and computational fluid dynamics. NMR Biomed 2009;22:795-808

38. van Ooij P, Guedon A, Poelma C, et al. Complex flow patterns in a real-size intracranial aneurysm phantom: phase contrast MRI compared with particle image velocimetry and computational fluid dynamics. NMR Biomed 2012;25:14-26

39. van Ooij P, Potters WV, Guédon A, et al. Wall shear stress estimated with phase contrast MRI in an in vitro and in vivo intracranial aneurysm. J Magn Reson Imaging 2013;38:876-84

40. Byun HS, Rhee K. CFD modeling of blood flow following coil embolization of aneurysms. Med Eng Phys 2004;26:755-61

41. Bradley WD, Walunch V. Blood flow: magnetic resonance imaging. Radiology 1985;154:443-50

42. Sevick RJ, Tsuruda JS, Schmalbrock P. Three-dimensional time-offlight MR angiography in the evaluation of cerebral aneurysms. J Comput Assist Tomogr 1990;14:874-81

43. Schuierer G, Huk WJ, Laub G. Magnetic resonance angiography of intracranial aneurysms: comparison with intra-arterial digital subtraction angiography. Neuroradiology 1992;35:50-54

44. Kim SE, Parker DL. Time-of-flight angiography. In: Carr JC, Carroll TJ, eds. Magnetic Resonance Angiography: Principles and Applications. Vol 39. New York: Springer-Verlag Science+Business Media; 2012 